\title{
PHYSICAL COMPONENT PERFORMANCE DEGRADATION DETECTION BASED ON ERROR PROBABILITY ANALYSIS
}

\author{
Barbara Walsh, Gerard Baldwin and Ronan Farrell \\ Centre for Telecommunications Value-Chain Research \\ Institute of Microelectronics and Wireless Systems \\ National University of Ireland \\ Maynooth \\ Ireland
}

\begin{abstract}
The detection and diagnosis of physical layer faults at system level in communications systems remains problematic. This paper presents a cross layer fault detection scheme, suitable for implementation at higher levels in a communications system, with the ability to detect and diagnose the cause of physical layer failure which results in impaired performance. It is based on the distribution of symbol error frequency and the probability of error of the underlying modulation scheme used in the communications system. With this knowledge, a simple counting algorithm is proposed as a diagnostic tool to implement the methodology.
\end{abstract}

\section{INTRODUCTION}

In modern communications systems much of the physical layer activity is hidden from the system behind several layers of abstraction. The typical base station is a good example of how this is achieved, as shown by the Open Base Station Architecture Initiative (OBSAI) model [1] seen in Fig.1. It is divided into the transport, baseband, radio frequency (RF) and clock and control blocks. As a signal passes through these blocks, it undergoes many stages, each of which can distort the signal. The RF module in particular can have a large impact on the signal as a result of functions such as digital to analog conversion, up-conversion, filtering, amplification and power combining. To further complicate matters, such base station modules in contemporary communications systems are constructed from various third party sub-systems. These components do not necessarily have built-in fault diagnosis interfaces and if they have, these interfaces are almost invariably proprietary and often prohibitively expensive.

The RF module is often the cause of system failure due to the challenging thermal conditions and high power loads to which it is subjected. The cost of system downtime for wireless service providers is extensive both in terms of lost revenue and diminishing customer confidence. Hence a cost effective method to predict system failure or degradation early is desirable as it would allow preemptive, noninvasive maintenance and reduced service interruption.

An alternate approach to using built-in fault diagnostics, based on the distribution of symbol error frequency, may be taken. It is well known that a certain proportion of the received symbols in such a communications system will be received erroneously. That is to say, symbol $\mathrm{X}$ has been transmitted but has been interpreted as symbol $\mathrm{Y}$ at the receiver. Under normal

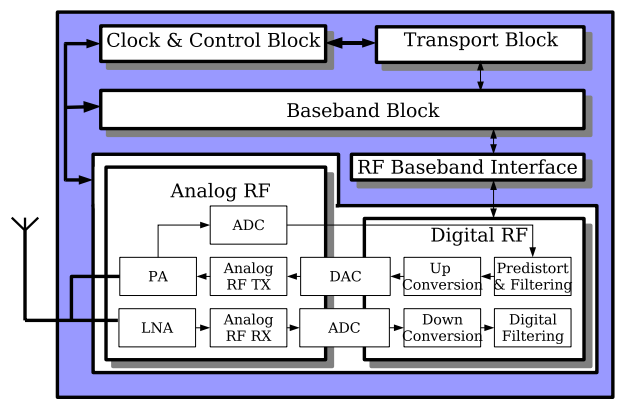

Figure 1: Base Station Infrastructure [1]

conditions the modulation scheme is designed to guarantee an equal probability of error for each symbol. However, with the addition of some nonlinearity, or other distorting factor, to the signal path this would no longer be the case. Instead these distortions would produce characteristic patterns in the symbol error ratios.

If a failing power amplifier (PA) is modeled as having degraded linearity performance, then it becomes possible to identify the on-set of this specific form of distortion in the data stream at the receiver and diagnose the existence of a failing PA in the signal chain. Since power amplifiers are quite prone to failure, a PA will be used as the test case for the fault diagnosis tool.

Using these ideas as the foundation of this work, Section II details the theoretical analysis for the particular case considered, 16-QAM. Equations are developed for the probability of error in the presence of AWGN and in the presence of AWGN with a failing PA, represented by a nonlinearity. The Matlab simulations, carried out to corroborate the theoretical calculations and to ensure that they bear a relationship to the real world situation, are briefly described in Section III. Numerical and simulated results are given in Section IV. The tool which arises from these results and a possible implementation utilizing a cross layer mechanism are described. Section $\mathrm{V}$ gives some conclusions.

\section{ERROR PROBABILITY}

We propose that the error probability for individual symbols is affected by nonlinear distortion and that the resulting error probabilities have a distinctive pattern associated with the par- 


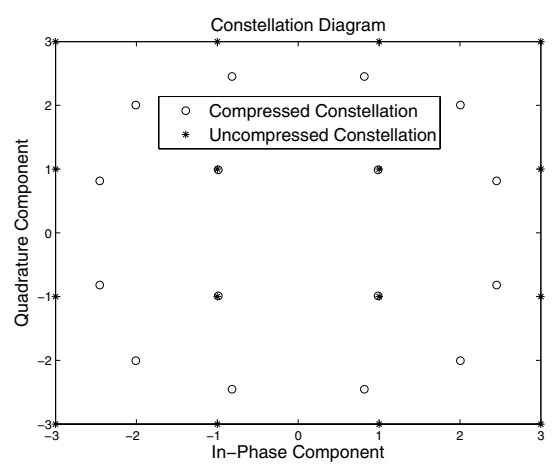

Figure 2: 16-QAM Constellation Diagrams

ticular form of distortion. In this section we prove this theory for the error probability of a power amplifier with gain compression.

The error probabilities for 16-QAM with an AWGN channel and 16-QAM with a failing PA and an AWGN channel are described in this section. In Fig. 2 the 16-QAM constellation diagram can be seen both before (uncompressed) and after (compressed) it has been subjected to the failing PA.

Since the error probability of the modulation format is used to validate simulation results, this section will include the theoretical analysis required to complete this validation procedure. M-QAM has been selected as the modulation scheme because it is a commonly used modulation format in modern communications systems.

\section{A. Error Probability for M-QAM with AWGN Channel}

A M-QAM constellation point $S_{N}$ is denoted by $\left(a_{N} \frac{d \min }{2}, b_{N} \frac{d_{\min }}{2}\right)$ where $d_{\min }$ is the minimum distance between the constellation points, $a_{N}$ and $b_{N}$ are integers, $\frac{d_{\min }}{2}=\sqrt{E_{O}}$ and $E_{O}$ is the energy of the symbol with the lowest amplitude. The M-QAM signal for symbol $k$ is given by [2] (p. 369):

$$
s_{k}(t)=\sqrt{\frac{2 E_{O}}{T}} a_{k} \cos 2 \pi f_{c} t-\sqrt{\frac{2 E_{O}}{T}} b_{k} \sin 2 \pi f_{c} t
$$

The probability of symbol error, $P_{M}$, with an AWGN channel is given by [3] (p. 278):

$$
P_{M}=1-\left(1-P_{L}\right)^{2}
$$

where $P_{L}$ is given by [2] (p. 371):

$$
P_{L}=2\left(1-\frac{1}{L}\right) Q\left(\sqrt{\frac{2 E_{O}}{N_{O}}}\right)
$$

$E_{O}$ is given by [2] (p. 372):

$$
E_{O}=\frac{3 E_{S}}{2\left(L^{2}-1\right)}
$$

Resulting in [3] (p. 278):

$$
P_{L}=2\left(1-\frac{1}{L}\right) Q\left(\sqrt{\frac{3}{L^{2}-1} \frac{E_{S}}{N_{O}}}\right)
$$

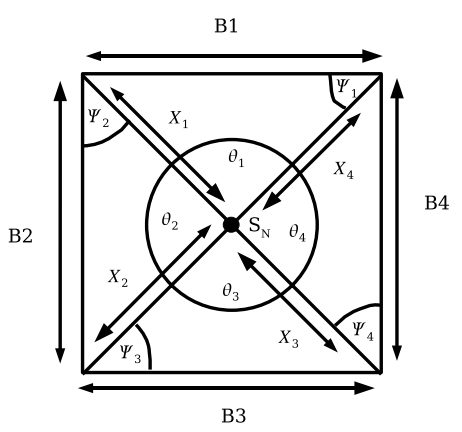

Figure 3: Signal Point and its Decision Boundary for 16-QAM with AWGN Channel

where $\frac{E_{S}}{N_{O}}$ is the signal to noise ratio (SNR) of the signal and $L=\sqrt{M}$.

The error probability of each individual M-QAM symbol, as opposed to the overall probability of symbol error, can be calculated numerically using the method proposed by Craig in [4] which may be summarized as follows. If an arbitrary M-QAM constellation symbol $S_{N}$ is transmitted, an error in reception occurs if the noise causes the receiver to produce a point outside the decision boundaries B1, B2, B3 or B4 as shown in Fig. 3 , otherwise it is received correctly. In the AWGN case, each of the sub-regions of the decision region shown in Fig. 3 are identical since ideally, the symbol is transmitted at the exact centre of the decision region. The probability that detected point lies outside a boundary is given by [4]:

$$
P_{S_{N}}=\frac{1}{\pi} \sum_{k=1}^{n} \int_{0}^{\theta_{k}} \exp \left[\frac{-X_{k}^{2} \sin ^{2}\left(\Psi_{k}\right)}{2 \sigma^{2} \sin ^{2}\left(\theta+\Psi_{k}\right)}\right] d \theta
$$

where $n$ is the number of sub-regions surrounding the constellation symbol, $\Psi_{k}$ and $\theta_{k}$ are the angles for each of the subregions shown in Fig. 3 and $X_{k}^{2} / 2 \sigma^{2}$ is a scaled version of the SNR. The scaling factor was found using the methodology proposed by Chrisikos in [5]. For any symbol, the number of terms in the sum $P_{S_{N}}$ will be equal to the number of sides on its decision region. The probability of symbol error is given by [4]:

$$
P_{S}=\sum_{n=1}^{N} P_{S_{N}} P\left(S_{N}\right)
$$

where $P\left(S_{N}\right)$ is the a priori probability that the symbol $S_{N}$ is transmitted.

\section{B. Error Probability for M-QAM with a Nonlinear PA and an AWGN Channel}

As was previously mentioned, the failing PA is modeled as having a degraded linearity performance. The error probability including PA nonlinearity cannot be found using standard error probability formulas such as (2) and so it is also calculated using the methodology proposed by [4]. However, the decision region is divided into different sub-regions than in the AWGN 


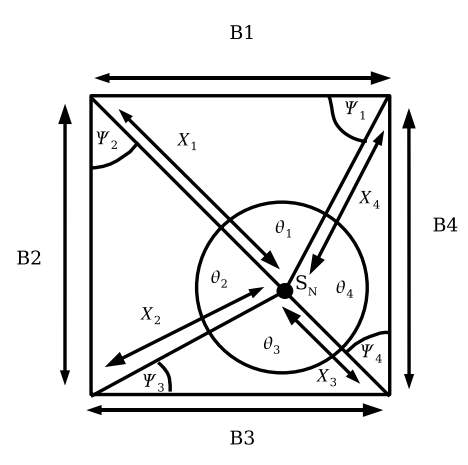

Figure 4: Signal Point and its Decision Boundary for 16-QAM with a Nonlinear PA and AWGN Channel

case. When the symbol is passed through a nonlinear PA before transmission, compression occurs, which results in the symbol moving away from the centre of the decision region. An example of an arbitrary M-QAM contellation symbol distorted by a nonlinear PA is shown in Fig. 4. Clearly these sub-regions are not identical and the required parameter values for (6) are given in Section V.

\section{Simulation}

Before considering the numerical results produced by the analysis expounded in Section II, simulations were carried out for the purposes of comparison.

These simulations were carried out in Matlab [6]. They computed computed the symbol error probability and symbol relative frequency distributions using the Communications Toolbox. 16-QAM modulation has been selected for these simulations. The $\frac{E_{B}}{N_{O}}$ of the channel is varied from $0 \mathrm{~dB}$ to $20 \mathrm{~dB}$ in the simulation. When simulating the nonlinear PA with AWGN channel the Rapp Model was used.

\section{A. Power Amplifier Model}

The PA was simulated using the Rapp Model. The AM/AM and AM/PM conversions were applied according to the following functions [7]:

$$
\begin{gathered}
F_{A M / A M}=\frac{u}{\left[1+\left(\frac{u}{v_{s a t}}\right)^{2 p}\right]^{\frac{1}{2 p}}} \\
F_{A M / P M}=0
\end{gathered}
$$

where $u$ is the magnitude of the input signal, $p$ is the smoothness factor and $v_{\text {sat }}$ is the maximum output amplitude.

\section{NumericAl Results}

\section{A. AWGN}

The probability of error for the simulated values was calculated as follows:

$$
P_{E}=\frac{\# S_{E}}{\# S_{R X}}
$$

where $\# S_{E}$ is the number of symbols received erroneously and $\# S_{R X}$ is the total number of symbols received. A graphical

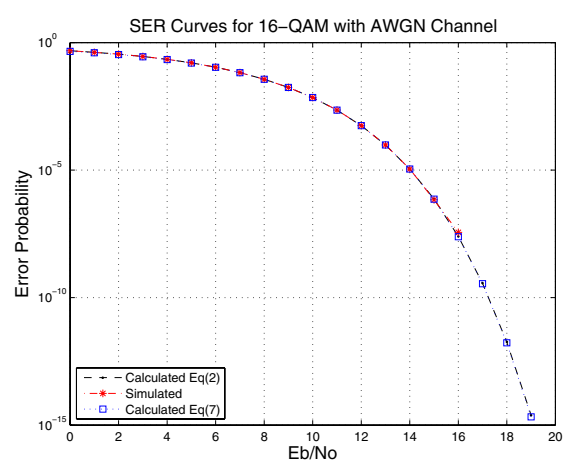

Figure 5: Symbol Error Curve for 16-QAM with AWGN Channel

comparison between the calculated values for the symbol error rate in the white noise environment, using (2) and (7), and the simulated values can be seen in Fig. 5. There is a very close match between simulated and calculated results in the case of white noise.

The probability of error for each constellation point was simulated and calculated using the methodology elucidated in [4] with $\theta_{k}=\pi / 2, \Psi_{k}=\pi / 4, x_{k}=\sqrt{2}$ and $B_{k}=2$. The value of $B_{k}$ depends only on the modulation format. These parameter values were calculated using the layout in Fig. 3. The use of elementary trigonometry results in the angles $\theta_{k}$ and $\Psi_{k}$ and the magnitude $x_{k}$, given foreknowledge of $B_{k}$ and the constellation symbol $S_{N}$. In the white noise case each of the 4 sub-regions of the decision region are identical. Depending on which constellation symbol is being considered, the total probability of error for that symbol will be the sum of the probability that the symbol falls outside either 2,3 or 4 of these sub-regions. The simulated error probability for each symbol was found as follows:

$$
P_{E_{i}}=\frac{\# S_{E_{i}}}{\# S_{R X_{i}}}
$$

where $\# S_{E_{i}}$ is the number of times symbol $i$ is received erroneously and $\# S_{R X_{i}}$ is the total number of times symbol $i$ is received. A graphical comparison of the calculated and simulated results can be seen in Fig. 6. As can be clearly discerned the calculated results replicate the simulated results quite closely.

\section{B. Nonlinear PA}

A graphical comparison between the calculated values for the symbol error rate for an AWGN channel with a nonlinear PA using (7) and the simulated values can be seen in Fig. 7. As can be seen, the simulted and calculated results are closely matched.

The error probability for each symbol is calculated using (6). Tables 1 and 2 contain the parameters, for a selection of constellation points with $v_{\text {sat }}=3 \mathrm{~V}$ and $v_{\text {sat }}=3.5 \mathrm{~V}$ respectively and $p=3$ in both cases, required by (6). These parameter values were calculated using the layout in Fig. 4. Once again, the use of elementary trigonometry results in the angles $\theta_{k}$ and $\Psi_{k}$ 


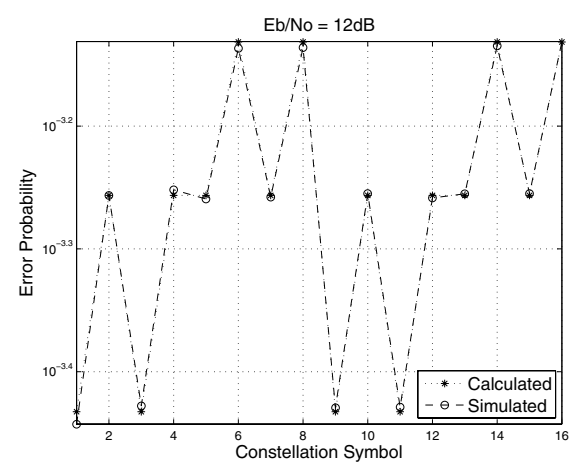

Figure 6: Error Probability for 16-QAM with an AWGN Channel for Each Constellation Symbol

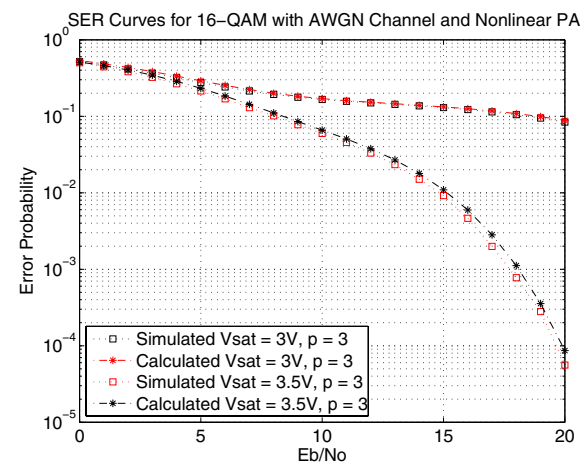

Figure 7: Symbol Error Curve for 16-QAM with an AWGN Channel with a Nonlinear PA

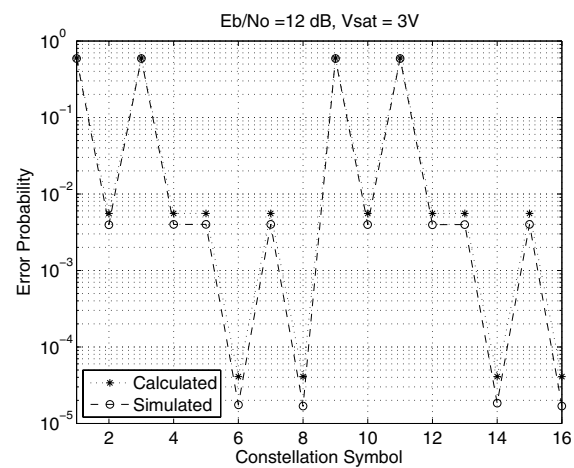

Figure 8: Error Probability for 16-QAM with Nonlinear PA and AWGN Channel for Each Constellation Symbol $\left(v_{\text {sat }}=3 \mathrm{~V}\right)$

and the magnitude $x_{k}$, given foreknowledge of $B_{k}$ and the constellation symbol $S_{N}$. These constellation points are in the first quadrant of the constellation diagram. The constellation points in the other 3 quadrants mirror these values as can be seen in Fig. 2. The error probability of each symbol is calculated using (11) for the simulated results. Graphical comparisons of the calculated and simulated results can be seen in Fig. 8 and Fig. 9 for $v_{\text {sat }}=3 \mathrm{~V}$ and $v_{\text {sat }}=3.5 \mathrm{~V}$ respectively and $p=3$ in both cases. As can be seen from these plots there is a close correlation between the simulated and calculated results.
Table 1: Parameter Values for (6) with a Nonlinear PA and AWGN Channel $\left(v_{\text {sat }}=3 \mathrm{~V}, \frac{E_{B}}{N_{0}}=12 \mathrm{~dB}\right)$

\begin{tabular}{|c||c|c|c|c|c|}
\hline$S_{N}$ & $\mathrm{k}$ & $\theta_{k}$ & $\Psi_{k}$ & $x_{k}$ & $B_{k}$ \\
\hline \hline 9 & 1 & 0.8271 & 0.7854 & 1.9216 & 2 \\
\hline 9 & 2 & 2.3145 & 0.0417 & 0.1133 & 2 \\
\hline 9 & 3 & 2.3145 & 0.7854 & 1.9216 & 2 \\
\hline 9 & 4 & 0.8271 & 1.5291 & 2.7152 & 2 \\
\hline \hline 13 & 1 & 1.2333 & 0.8907 & 1.6480 & 2 \\
\hline 13 & 2 & 1.6218 & 0.5532 & 1.0523 & 2 \\
\hline 13 & 3 & 2.0522 & 0.6042 & 1.2820 & 2 \\
\hline 13 & 4 & 1.3759 & 1.0857 & 1.8034 & 2 \\
\hline \hline 14 & 1 & 1.5690 & 0.7854 & 1.4142 & 2 \\
\hline 14 & 2 & 1.5726 & 0.7836 & 1.4116 & 2 \\
\hline 14 & 3 & 1.5726 & 0.7854 & 1.4142 & 2 \\
\hline 14 & 4 & 1.5690 & 0.7872 & 1.4168 & 2 \\
\hline
\end{tabular}

Table 2: Parameter Values for (6) with a Nonlinear PA and AWGN Channel $\left(v_{\text {sat }}=3.5 \mathrm{~V}, \frac{E_{B}}{N_{0}}=12 \mathrm{~dB}\right)$

\begin{tabular}{|c||c|c|c|c|c|}
\hline$S_{N}$ & $\mathrm{k}$ & $\theta_{k}$ & $\Psi_{k}$ & $x_{k}$ & $B_{k}$ \\
\hline \hline 9 & 1 & 1.0046 & 0.7854 & 1.6757 & 2 \\
\hline 9 & 2 & 2.1370 & 0.2192 & 0.5153 & 2 \\
\hline 9 & 3 & 2.1370 & 0.7854 & 1.6757 & 2 \\
\hline 9 & 4 & 1.0046 & 1.3516 & 2.3131 & 2 \\
\hline \hline 13 & 1 & 1.3798 & 0.8466 & 1.5258 & 2 \\
\hline 13 & 2 & 1.6196 & 0.6556 & 1.2207 & 2 \\
\hline 13 & 3 & 1.8008 & 0.7044 & 1.3302 & 2 \\
\hline 13 & 4 & 1.4830 & 0.9344 & 1.6148 & 2 \\
\hline \hline 14 & 1 & 1.5701 & 0.7854 & 1.4142 & 2 \\
\hline 14 & 2 & 1.5715 & 0.7847 & 1.4132 & 2 \\
\hline 14 & 3 & 1.5715 & 0.7854 & 1.4142 & 2 \\
\hline 14 & 4 & 1.5701 & 0.7861 & 1.4152 & 2 \\
\hline
\end{tabular}

\section{Fault Diagnosis Mechanism}

As the error probability for both an AWGN channel and a nonlinear PA with AWGN channel have been simulated and the results have been verified theoretically, the applicability of relative frequency distributions in the detection of component performance degradation can now be considered.

The relative frequency is the principle upon which the counting algorithm for the proposed fault detection tool is based, and is computed using the following formula:

$$
F_{R}=\frac{\# S_{X}}{\# S_{R X}}
$$

where $\# S_{X}$ is the number of times a particular symbol is re- 


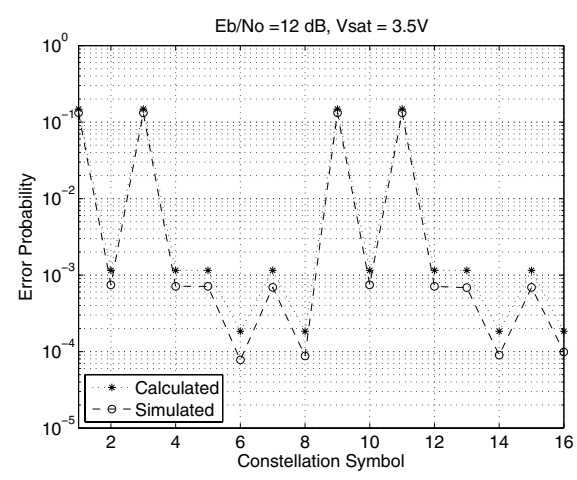

Figure 9: Error Probability for 16-QAM with Nonlinear PA and AWGN Channel for Each Constellation Symbol $\left(v_{\text {sat }}=3.5 \mathrm{~V}\right)$

ceived and $\# S_{R X}$ is the total number of symbols received. Hence, it would be necessary only to count the total number of symbols received and the total number of each different symbol received to implement the counting algorithm and fault diagnosis mechanism.

The relative frequency for the AWGN channel simulation was generated and is shown in Fig. 10. A clear pattern can be seen in these circumstances. Each constellation point has virtually the same relative frequency $\left(F_{R}=\frac{1}{16}\right)$ which is irrespective of the values $\frac{E_{B}}{N_{0}}$. The nonlinear PA with AWGN channel simulation yields the relative frequency plots which can also be seen in Fig. 10. In this instance there is also a very clear pattern which is followed over the range of $\frac{E_{B}}{N_{0}}$. The symbols fall into three distinct categories depending on the level of compression to which they are subjected. Moreover, the value of the relative frequency is affected by the level of nonlinearity and hence can be used to detect a change in the PA performance over time.When the relative frequency curve for the AWGN channel is contrasted with a nonlinear PA with AWGN channel the resulting distributions are distinctively dissimilar. Ergo, the source of degradation can be identified as gain compression with great facility on this occasion.

It is proposed that a similar methodology to that which has been described in this work be used to generate symbol error frequency distributions for other fault mechanisms which may arise within the RF module of a wireless system. These distributions would be stored in a database. The symbol error frequency distribution of a system functioning correctly would be compared to the system symbol error frequency distribution at regular intervals. If the system symbol error frequency distribution does not match the symbol error frequency distribution of a system functioning correctly, the distribution is compared to those stored in the database. The fault detection mechanism thereby detects any undesirable system behaviour on-line and may possibly provide a diagnosis for the fault.

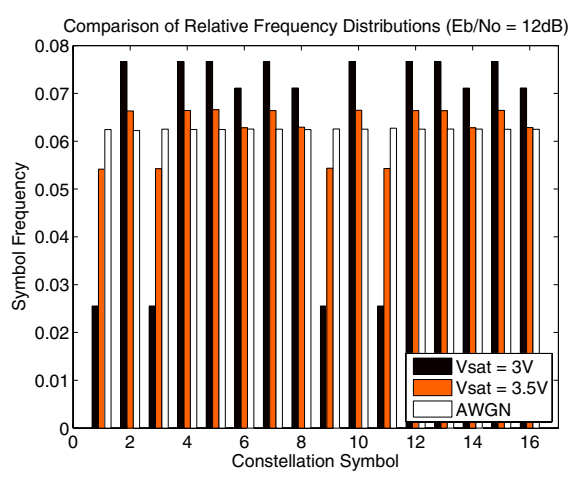

Figure 10: Comparison of Relative Frequency for Each Constellation Point for an AWGN Channel and a Nonlinear PA with AWGN Channel $\left(\frac{E_{B}}{N_{0}}=12 \mathrm{~dB}\right)$

\section{CONCLUSION}

The approach presented in this paper is useful in the area of physical layer fault detection. The effect of component degradation on individual symbol error rates due to a failing PA is simulated and verified theoretically. The source of the deterioration in system performance can be identified using the relative frequency distribution for a nonlinear PA because its distribution has a characteristic pattern. Moreover, a cross layer diagnostic tool to monitor the symbol relative frequency distribution, based on the aforementioned counting algorithm, could easily be implemented to detect any such deviant behaviour. Due to the computational efficiency of this algorithm, it may be implemented on-line in a live communications system.

\section{ACKNOWLEDGMENT}

The authors would like to thank the Centre for Telecommunications Value-Chain Research (CTVR) and the Science Foundation of Ireland (SFI) for supporting this research.

\section{REFERENCES}

[1] OBSAI, "Open Base Station Architecture Initiative BTS System Reference Document Version 1.0”, OBSAI, 2002.

[2] S. Haykin, Communication Systems, 4th ed. New York, 2001.

[3] J. G. Proakis, Digital Communications, 4th ed. New York, 2001.

[4] J. W. Craig, "A New, Simple Exact Result for Calculating the Probability of Error for Two-Dimensional Signal Constellations", Proceedings of the IEEE Military Communications Conference, 1991, vol.2, pp:II571 II575.

[5] G. Chrisikos, "Analysis of 16-QAM Over a Nonlinear Channel", Proceedings of the Ninth IEEE International Symposium on Personal, Indoor and Mobile Radio Communications, 1998, vol. 3, pp:1325 - 1329

[6] http://www.mathworks.com/, version 7.2.0.294 (R2006a), January 27, 2006

[7] C. Rapp, "Effects of HPA-Nonlinearity on a 4-DPSK/OFDM-Signal for a Digitial Sound Broadcasting System", Proceedings of the Second European Conference on Satellite Communications, 1991, pp. 179-184 